\title{
Isolation and Some Properties of the Competence Factor from Group H Streptococcus Strain CHALlis
}

\author{
By W. T. DOBRZANSKI AND H. OSOWIECKI \\ Department of Microbiology and Hygiene, University Medical School, \\ Warsaw, Poland
}

(Accepted for publication II October 1966)

\begin{abstract}
SUMMARY
In several bacterial species the occurrence of competence in transformation is strictly related to the production of a competence factor (c.f.). In the present work this factor was isolated from the cell surface of Streptococcus group $\mathrm{H}$ strain CHALLIS by the centrifugation of competent bacteria at $\mathrm{pH} 2 \cdot 0$, followed by thermal shock. Streptococci of strain WICKY adsorb c.f. isolated from the cHALLIS organisms because supernatant fluids containing c.f. lose their activity after having been in contact with organisms. It was possible to 'tear off' again from such organisms of strain wICKY the c.f. they had adsorbed. The re-isolated c.f. was still active in the transformation systems used.

Some biochemical properties of the c.f. from strain CHALLIS are described. The factor was relatively heat resistant and sensitive to proteolytic enzymes. Streptococcal c.f. or a fragment of it seems to be a protein or polypeptide of fairly low molecular weight. This streptococcal c.f. is probably different from the pneumococcal c.f.
\end{abstract}

\section{INTRODUCTION}

The incorporation of DNA in bacterial transformation depends on the occurrence in recipient cells of a transient competence state. The elucidation of the mechanism of competence-that is to say, the mechanism of incorporation of DNA-is a biologically important problem.

Recently it has been shown that the appearance of competence is strictly related to the production by the cells of a special competence factor (c.f.) (Pakula \& Walczak, 1963; Felkner \& Wyss, 1964; Tomasz \& Hotchkiss, 1964; Charpak \& Dedonder, I965; Tomasz \& Mosser, I966).

In Streptococcus group H strain CHALlis Pakula \& Walczak (1963) discovered and observed c.f. in the supernatants of cultures after the cells had excreted it into a rich, undefined medium, already containing several different polypeptides. Under conditions which produce competence and transformation of other streptococci and of pneumococci it is impossible to get a competent population and thus transformation in Streptococcus strain wiCKY (Pakula, 1965). This strain can be transformed only in the presence of the c.f. produced by strain CHALLis.

It was recently reported that it is possible 'to tear off' the c.f. from the cell surface of Bacillus subtilis and pneumococci. Some biochemical properties of the both factors have been described (Charpak \& Dedonder, 1965; Tomasz \& Mosser, 1966).

In the present work, the c.f. from the surface of cocci of strain CHALEIS was isolated and a preliminary biochemical characterization made. 


\section{METHODS}

Bacterial strains. The transformable strain CHALLIS of Streptococcus group $\mathbf{H}$, sensitive to streptomycin, was used to obtain the competence factor. A mutant of strain CHALLIS resistant to $2 \mathrm{mg}$. streptomycin/ml. served as donor of transforming DNA. Streptococcus group H strain WICKY was used as recipient. Both these strains originated from Dr Pakula's collection.

DNA preparation. DNA was extracted from strain cHALLIs cultures by a method described in Pakula, Piechowska, Bankowska \& Walczak (1962). This method involved lysis of cells by the lytic factor from a culture of Streptomyces albus and isolation and purification of the DNA according to the procedure elaborated by Marmur (196I).

Media. For the production of c.f. by strain CHALLIS and for the transformation of strain wICKY, two media employed by Pakula \& Walczak (1963) and Pakula (1965) were used: ET 3 (I \% Difco Neopeptone, $0.5 \%$ Difco yeast extract, $0.5 \% \mathrm{NaCl}, 0.17 \%$ $\mathrm{K}_{2} \mathrm{HPO}_{4}$ and $0.2 \%$ glucose in distilled water) and HE, which differed from ET 3 by the replacement of water with beef heart extract. The $\mathrm{pH}$ of both media was adjusted to $7 \cdot 6$. The media were sterilized by filtration through a glass filter G-5 or through a Seitz filter. Albumin $(0.2 \%)$ was always added to both media to obtain c.f. from strain CHALLIS and transformation in strain WICKY.

Transformation procedure and measurement of the activity of the competence factor An overnight culture of strain wICKY in horse blood broth was inoculated at $4 \times 10^{6} \mathrm{cocci} / \mathrm{ml}$. into $\mathrm{ET}_{3}$ or $\mathrm{HE}$ medium and incubated for $90 \mathrm{~min}$. at $37^{\circ}$ in a water bath. After this period, $\mathrm{I} \cdot 75 \mathrm{ml}$. of the culture was supplemented with $0.5 \mathrm{ml}$. of a suitable dilution of treated and untreated c.f. (first positive control) and incubated for $30 \mathrm{~min}$. at $37^{\circ}$. At this time, $0.25 \mathrm{ml}$. of transforming DNA (Io $\mu \mathrm{g}$./ $/ \mathrm{ml}$.) was added to the mixture and incubated $15 \mathrm{~min}$., then deoxyribonuclease ('Distreptaza'-about $20 \mathrm{u}$. $/ \mathrm{ml}$.) was added to stop the reaction. Afterwards the culture was incubated for an additional $120 \mathrm{~min}$. for phenotypic expression of the transformed marker.

Immediately after the addition of DNA, a count was made of the number of viable colony-forming units. After phenotypic expression, the number of streptomycinresistant transformants was determined on blood agar plates containing $250 \mu \mathrm{g}$. $/ \mathrm{ml}$. of streptomycin.

As a second positive control, the transformation of strain wICKY provoked by Pakula's type of supernatant containing c.f. (Pakula \& Walczak, 1963) was performed in each experiment. As a negative control in each experiment, physiological saline was added to the reaction mixture in place of c.f.

The method of 'tearing off' c.f. is described below. Each sample of c.f., before being used in the experiments, was characterized by the determination of the yield of a transformation of strain wICKY performed in the presence of controlled c.f. in I0 $\%$ (v/v) concentration.

Reagents. Dihydrostreptomycin sulphate ('Polfa'), deoxyribonuclease ('Distreptaza'-'Biomed'), pronase ('Calbiochem'), papain ('Dembach'), trypsin ('Merck'), pancreatin ('Calbiochem'), lyso zyme (' Reanal') and ribonuclease ('BDH') were used. 
Preparation of competence factor.

An overnight culture of strain chaLlis in horse blood broth was inoculated at $4.3 \times 10^{6} \mathrm{cocci} / \mathrm{ml}$. into a quantity (usually 21 .) of $\mathrm{ET}_{3}$ or HE medium with albumin. The culture was incubated, usually for $100 \mathrm{~min}$., in a water bath at $37^{\circ}$. Each batch of

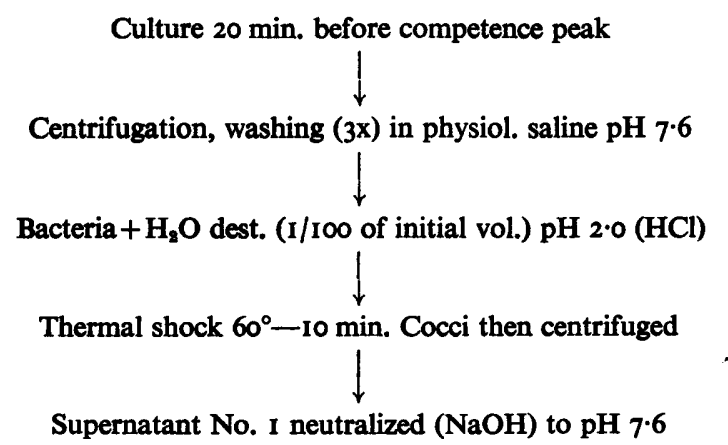

Fig. I. Method of 'tearing off' c.f. from Streptococcus strain cHALLIs. An amount of ET 3 or HE medium containing $0.2 \%$ albumin was inoculated with an overnight culture of the strain challs in horse blood broth. At the beginning of the incubation the medium contained about $4.3 \times 10^{8} \mathrm{cocci} / \mathrm{ml}$. The culture was incubated for a suitable period of time (usually $100 \mathrm{~min}$.) in a water bath at $37^{\circ}$.

ET 3 or HE medium used for the preparation of c.f. was thoroughly tested, by determining the time at which the peak of the competence occurred. Further details of the procedure for 'tearing off' c.f. from strain CHALLIs devised during the course of the present study are shown in Fig. I.

We did not succeed in isolating the strain cHALLIs c.f. by the method used for pneumococci by centrifugation in physiological saline + phosphate buffer +2 -mercaptoethanol (pH 7.5) (Tomasz \& Mosser, 1966).

\section{Optimal period of contact.}

The supernatant fraction of media containing streptococcal c.f. obtained by Pakula \& Walczak (1963) requires about $30 \mathrm{~min}$. of contact with strain WICKY cells to produce the maximum yield of transformation. To find the optimum time of contact for the c.f. prepared according to the method described above, I0 \% of 'torn off' c.f. was added to samples taken from the same culture of strain wiCKY. After incubation for the times as indicated in Fig. 2, the colony count and the number of transformants were determined.

Figure 2 shows that the optimal time of contact between the c.f. and strain wICKY is also about $30 \mathrm{~min}$.

\section{Effect of competence factor concentration on yield of transformants.}

To test the relationship between transformation yield and the concentration of c.f. added to the recipient cells in a transforming medium, $0.5 \mathrm{ml}$. of phosphate buffer containing various concentrations of c.f. was added to samples of the same culture of strain WICKY, and after $30 \mathrm{~min}$. incubation the number of transformants was determined.

Figure 3 presents the results of such an experiment with the use of one of our 
supernatants no. I (see Fig. I) as c.f. Using this c.f. 10 \%(v/v) concentration of the factor was saturating. This concentration was saturating in the majority of our preparations, although in some preparations 15 or $20 \%(\mathrm{v} / \mathrm{v})$ of c.f. was required to reach saturation:

\title{
Effect of proteolytic enzymes.
}

Sensitivity of the competence factor to various enzymes was studied to obtain some information about the chemical nature of the factor.

In these experiments, presented in Table $\mathrm{I}, 0.4 \mathrm{ml}$. of c.f. was incubated at $37^{\circ}$ with $0.1 \mathrm{ml}$. of the given enzyme at the desired concentration for the period of time indicated. Then I.75 ml. of strain wICKY culture prepared in the usual manner was added to this sample and incubated for $30 \mathrm{~min}$. at $37^{\circ}$. The remaining c.f. activity was assayed

Table I. Enzyme sensitivity of competence factor from Streptococcus strain CHALLIS

\begin{abstract}
$0.4 \mathrm{ml}$. of $\mathrm{c} \mathrm{f}$. was incubated with $0.1 \mathrm{ml}$. of the given enzyme in desired concentration at $37^{\circ}$ for the indicated period of time. Then $1.75 \mathrm{ml}$. of strain wICKY culture prepared in the usual manner was added to this sample and incubated for $30 \mathrm{~min}$. at $37^{\circ}$. Afterwards a transformation was performed as described under Methods.
\end{abstract}

\begin{tabular}{|c|c|c|c|c|}
\hline \multirow[b]{2}{*}{ Enzyme } & \multirow{2}{*}{$\begin{array}{c}\text { Concentra- } \\
\text { tion } \\
(\mu \mathrm{g} . / \mathrm{ml} .)\end{array}$} & \multicolumn{3}{|c|}{$\begin{array}{l}\text { Percentage of residual c.f. activity } \\
\text { after treatment }\end{array}$} \\
\hline & & $10 \mathrm{~min}$. & $90 \mathrm{~min}$. & $180 \mathrm{~min}$ \\
\hline \multirow{3}{*}{ Pronase } & I & I $\cdot 0$ & - & - \\
\hline & 10 & - & 0 & $\overline{0}$ \\
\hline & 100 & - & - & 0 \\
\hline \multirow{3}{*}{ Trypsin } & I & 63 & - & - \\
\hline & 10 & - & $5 \cdot 0$ & - \\
\hline & 100 & - & $I \cdot 7$ & 0 \\
\hline \multirow{3}{*}{ Pancreatin } & I & 100 & - & - \\
\hline & 10 & - & 14.7 & - \\
\hline & 100 & - & 0.25 & 0.4 \\
\hline \multirow{4}{*}{ Papain } & I & 100 & - & - \\
\hline & IO & - & $3 \cdot 0$ & - \\
\hline & 100 & - & 0 & 0.4 \\
\hline & & \multicolumn{2}{|c|}{$10 \mathrm{~min}}$. & $30 \mathrm{~min}$. \\
\hline \multirow{4}{*}{ Lysozyme } & $\mathbf{I}$ & \multicolumn{2}{|c|}{100} & - \\
\hline & 5 & \multirow{2}{*}{\multicolumn{2}{|c|}{100}} & - \\
\hline & 10 & & & 100 \\
\hline & I & \multicolumn{2}{|c|}{-} & - \\
\hline \multirow[t]{2}{*}{ RNAse } & 5 & \multirow{2}{*}{\multicolumn{2}{|c|}{100}} & - \\
\hline & 10 & & & 100 \\
\hline
\end{tabular}

by transformation of strain wICKY as described previously. Thus, during the period of treatment with c.f. and transformation, an enzyme was present, but in a concentration five times lower than that at the beginning of the experiment. To test if the decrease in the yield of transformants demonstrated in Table I was indeed due to enzymatic destruction of c.f. during incubation of the factor with the enzyme before treatment and transformation, special control experiments were carried out. In these experiments, a $30 \mathrm{~min}$. exposure of strain wICKY to c.f. was performed in the presence of $10 \mu \mathrm{g} . / \mathrm{ml}$. of trypsin, papain, or pancreatin. After this period, the cells were centrifuged, resuspended in fresh transformation medium and transformation was 
carried out. The yield of transformants in these experiments was normal or only slightly lower (about $10 \%$ ) than in positive controls treated in the same manner. The results of these experiments justify the conclusion that the streptococcal c.f. is sensitive to proteolytic enzymes. Termination of trypsin treatment of c.f. by the addition of soybean trypsin inhibitor does not prevent inhibition of activation in the case of similar observations on pneumococcal competence factor (Tomasz \& Mosser, 1966).

After Io min. of contact between c.f. and the enzymes at a concentration of I $\mu \mathrm{g} . / \mathrm{ml}$., only pronase destroyed the activity of the factor. Trypsin decreased its activity up to about $35 \%$, while pancreatin and papain were not active in these conditions. Ninety minutes contact between c.f. and the enzymes at a concentration of $10 \mu \mathrm{g} . / \mathrm{ml}$. caused almost complete destruction of c.f. activity. The pneumococcal factor is also very sensitive to proteolytic enzymes (Tomasz \& Mosser, 1966). Subtilisin, chymotrypsin, and trypsin at a concentration of I $\mu \mathrm{g}$. $/ \mathrm{ml}$. inactivate it completely after $10 \mathrm{~min}$. This difference indicates that the two factors differ in enzyme sensitivity. Lysozyme and ribonuclease do not inactivate either of the competence factors.

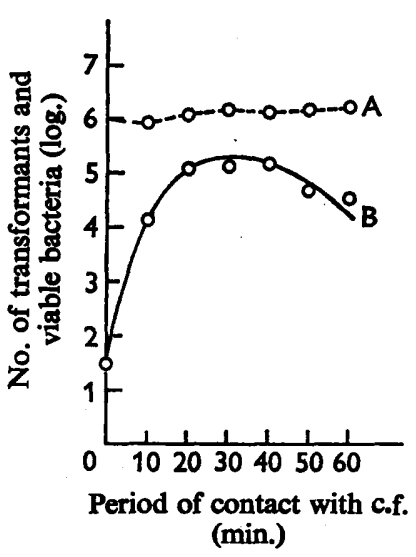

Fig. 2

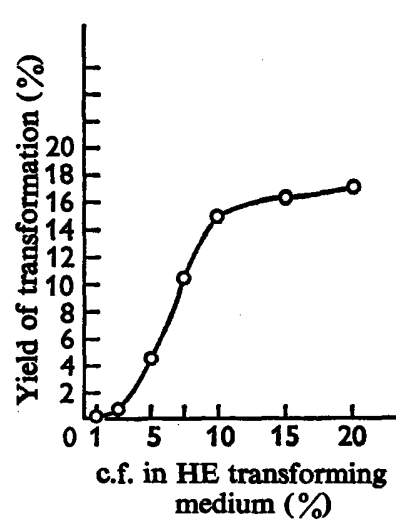

Fig. 3

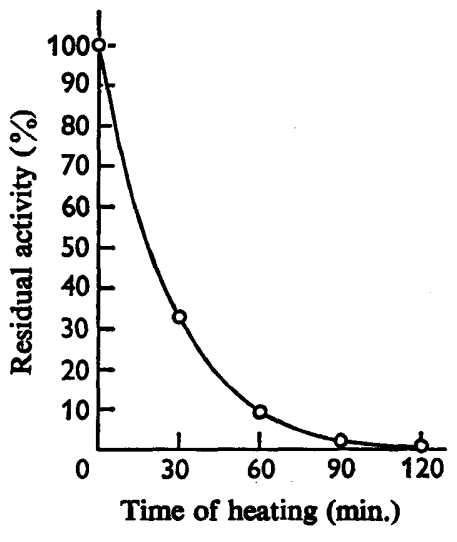

Fig. 4

Fig. 2. Optimal period of contact (before DNA addition) of strain WICKY with competence factor 'torn off' from cell surface: A, number of viable bacteria (log.); B, number of transformants (log.). To the samples taken from the same culture of strain wickY, the c.f. $10 \%(v / v)$ was added. Then, all samples were incubated for different periods of time. After the periods indicated in the figure, the numbers of viable centres and transformants were determined.

Fig. 3. Dependence of yield of reaction (\%) in strain WCKY on c.f. concentration in HE transforming medium. To the samples of the same culture of strain wICKY, $0.5 \mathrm{ml}$. of phosphate buffer containing various concentrations of c.f. was added and after $30 \mathrm{~min}$. incubation and transformation the yield of reaction was determined.

Fig. 4. Sensitivity to temperature $\left(100^{\circ}\right)$ of c.f. from strain challis. An amount of nondiluted c.f. was heated to $100^{\circ}$. After the periods indicated in the figure the samples of c.f. were taken, immediately diluted to $7.5 \%(v / v)$ and added to the recipient cells. Then, a transformation reaction was performed. The concentration of c.f. used in these experiments was a non-saturating one.

\section{Heat-inactivation of the competence factor}

The purpose of this experiment was to compare the heat-sensitivity of our c.f. with the sensitivity of streptococcal c.f. reported by Pakula \& Walczak (1963), pneumococcal c.f. (Tomasz \& Mosser, 1966) and Bacillus subtilis c.f. (Charpak \& Dedonder, 1965). 
In this experiment, a non-saturating concentration (i.e. $7.5 \%, v / v)$ of heated c.f. was used to evoke transformation. Undiluted c.f. was heated to $100^{\circ}$ and after the times indicated in Fig. 4 samples were taken, immediately diluted to the abovementioned non-saturating concentration and added to the recipient cells and its activity assayed by the transformation method. The results were compared with the result of a control experiment performed in parallel in which an unheated, suitably diluted sample of the same c.f. was used.

The results in Fig. 4 show that the factor was relatively heat-resistant: Io min. exposure to $100^{\circ}$ resulted in the loss of only about $22 \%$ of its activity. Complete loss of activity only occurred after $90 \mathrm{~min}$. heating at $100^{\circ}$. The c.f. from strain CHALLIS is much more heat-resistant than pneumococcal c.f., which lost all its activity after $20 \mathrm{~min}$. at $100^{\circ}$ (Tomasz \& Mosser, 1966). The c.f. from the Marburg strain of Bacillus subtilis is also much more heat-sensitive. It lost complete activity after $5 \mathrm{~min}$. at $100^{\circ}$ (Charpak \& Dedonder, 1965).

At $-15^{\circ}$, the streptococcal factor conserved activity for 5-7 days. Alkalization and storage in alkali at $\mathrm{pH}$ II prolongs activity up to about 20 days.

From these experiments we may conclude, from its relative heat resistance, that (I) streptococcal c.f. is or contains a protein or polypeptide of low molecular weight, and (2) that streptococcal c.f. is probably different from pneumococcal c.f.

We would like to thank Professor D. Shugar and Professor P. Szafranski for valuable discussion during the preparation of this manuscript, and $J$. Nalecz for excellent technical assistance.

\section{REFEREN CES}

Charpak, M. \& Dedonder, R. (1965). Production d'un 'facteur de competence' soluble par Bacillus subtilis Marburg ind 168. C. r. hebd. Séanc. Acad. Sci., Paris. 260, 5638.

FELKNER, I. C. \& Wyss, O. (1964). A substance produced by competent Bacillus cereus 569 cells that affects transformability. Biochim. biophys. Res. Comm. 16, 94.

MARMUR, J. (1961). A procedure for isolation of deoxyribonucleic acid from micro-organisms. $J$. molec. Biol. 3, 208.

PaKula, R. (1965). Production of competence-provoking factor and development of competence of a transformable Streptococcus in serum-free media. Can. J. Microbiol. Ir. 8I I.

Pakula, R. \& Walczak, W. (1963). On the nature of competence of transformable streptococci. J. gen. Microbiol. 31, 125 .

Pakula, R., Piechowska, M., Bankowska, E. \& Walczak, W. (1962). A characteristic of DNA mediated transformation system of two streptococcal strains. Acta microbiol. pol. Ir, 205.

Tomasz, A. \& Hotchisiss, R. D. (1964). Regulation of the transformability of pneumococcal cultures by macromolecular cell products. Proc. natn. Acad. Sci. U.S.A. 51, 480.

Tomasz, A. \& Mosser, J. L. (1966). On the nature of the pneumococcal activator substance. Proc. natn. Acad. Sci., U.S.A. 55, 58. 